\title{
Una documentazione al servizio delle occorrenze cliniche
}

\section{Health record systems that meet clinical needs}

\section{Gabriella Negrini*}

\author{
Direttore UOC Ospedali Area Ovest, Azienda USL Bologna
}

Ricevuto il 7 luglio 2011; accettato il 5 settembre 2011

disponibile online il 9 dicembre 2011

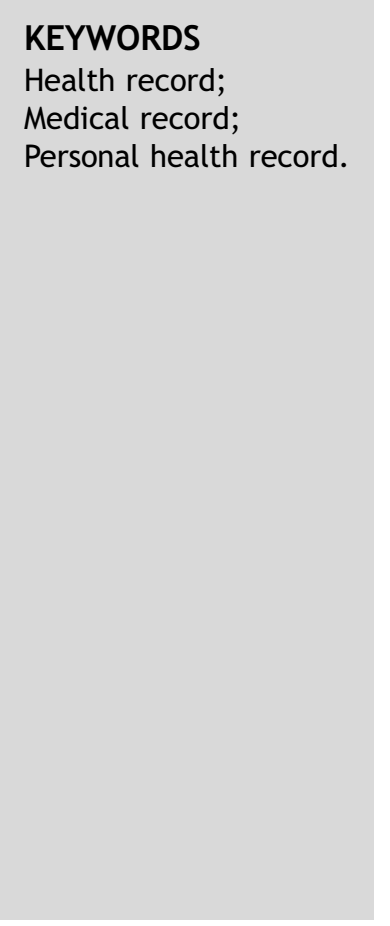

\begin{abstract}
Summary
Introduction: Increased attention has recently been focused on health record systems as a result of accreditation programs, a growing emphasis on patient safety, and the increase in lawsuits involving allegations of malpractice. Health-care professionals frequently express dissatisfaction with the health record systems and complain that the data included are neither informative nor useful for clinical decision making. This article reviews the main objectives of a hospital health record system, with emphasis on its roles in communication and exchange among clinicians, patient safety, and continuity of care, and asks whether current systems have responded to the recent changes in the Italian health-care system.

Discussion: If health records are to meet the expectations of all health professionals, the overall information need must be carefully analyzed, a common data set must be created, and essential specialist contributions must be defined. Working with health-care professionals, the hospital management should define how clinical information is to be displayed and organized, identify a functionally optimal layout, define the characteristics of ongoing patient assessment in terms of who will be responsible for these activities and how often they will be performed. Internet technology can facilitate data retrieval and meet the general requirements of a paper-based health record system, but it must also ensure focus on clinical information, business continuity, integrity, security, and privacy.

Conclusions: The current health records system needs to be thoroughly revised to increase its accessibility, streamline the work of health-care professionals who consult it, and render it more useful for clinical decision making - a challenging task that will require the active involvement of the many professional classes involved.

(c) 2011 Elsevier Srl. All rights reserved.
\end{abstract}

\footnotetext{
* Corrispondenza: Azienda USL Bologna, l.go Nigrisoli 2 - 40133 Bologna.

E-mail: gabriella.negrini@ausl.bo.it (G. Negrini).
} 


\section{Introduzione}

Negli ultimi decenni si è assistito a cambiamenti sostanziali nell'organizzazione dei servizi sanitari, nel nostro come in molti altri Paesi occidentali, e la trasformazione è tutt'altro che esaurita. Sotto l'impulso di nuove esigenze determinate da variazioni:

- nelle caratteristiche della popolazione da assistere (riduzione della natalità, maggiore consistenza delle fasce di età avanzata, composizione multietnica),

- nell'epidemiologia, con marcata prevalenza di patologie di tipo cronico,

- nella risorse economiche assegnate al settore,

- nelle conoscenze e nelle disponibilità tecnologiche,

- nelle attese degli utenti,

- nell'assetto delle professioni sanitarie,

- sono state avviati processi di ristrutturazione che hanno pesantemente coinvolto anche il complesso e variegato universo ospedaliero.

In un turbinio di nuovi assetti, nuovi modelli organizzativi, nuove figure sanitarie assurte al rango di professionisti, nuove tecniche di trattamento, nuovi percorsi di cura attraversanti diversi setting assistenziali, che cosa ne è stato della documentazione sanitaria?

La plurisecolare cartella clinica è stata oggetto di evoluzione funzionale rispetto a quanto andava modificandosi?

È constatazione comune che negli anni più recenti la cartella di ricovero sia stata oggetto di attenzioni forse mai riservate a essa in precedenza, complici i percorsi di accreditamento e di certificazione di qualità che hanno imposto requisiti ineludibili, il diffondersi della cultura della sicurezza clinica, ma anche le preoccupazioni indotte dal lievitare del contenzioso [1].

\section{Discussione}

Le principali funzioni che il fascicolo di ricovero dovrebbe assolvere, come nel tempo espresse concordemente da più autori, possono così compendiarsi [2-13]:

- clinico-assistenziale: attraverso l'esposizione dei problemi di salute del paziente, l'annotazione dei trattamenti diagnostici, terapeutici e delle attività assistenziali, si pone come uno strumento di ausilio alle comunicazioni tra $i$ sanitari, per migliorarne il raccordo operativo, facilitarne le decisioni e garantire la continuità delle cure;

- scientifico-didattico: di supporto ad audit, studi, attività di formazione e aggiornamento;

- giuridico-amministrativo: a tutela dei diritti e degli interessi legittimi degli assistiti, dell'ospedale e dei suoi operatori e per valutazioni sulle modalità e i costi dei servizi erogati.

L'odierna documentazione di un ricovero, peraltro, è sempre più multimediale, così che lo stesso fascicolo rappresenta solo una porzione di un agglomerato informativo frequentemente ben più corposo [14].

Oltreconfine si ritrovano inquadramenti non dissimili.

In Francia, dove esiste una disciplina nazionale della documentazione sanitaria, con fonte normativa primaria contenuta nel Code de la Santé Publique [15] e regolamentazione di dettaglio emanata dall'Agence Nationale d'Accréditation et d'Evaluation en Santé, poi confluita nella Haute Autorité de Santé, il dossier clinico - unico, con contributi apportati da professionisti di differente estrazione - deve perseguire le seguenti finalità: rendere disponibili le informazioni necessarie alla cura dell'assistito; mantenere traccia dei trattamenti eseguiti e delle decisioni prese; garantire la continuità delle cure; valutare la qualità clinico-assistenziale; favorire l'insegnamento e la ricerca; permettere l'estrazione dei dati necessari per l'analisi medico-economica e il controllo di qualità; fungere da supporto nei casi di supposta responsabilità.

La Joint Commission International, nel suo manuale per l'accreditamento degli ospedali [16], allo standard $\mathrm{MCl} .19 .1$ stabilisce che la cartella clinica deve contenere "informazioni sufficienti a identificare il paziente, supportare la diagnosi, giustificare il trattamento, documentare il decorso e i risultati del trattamento e promuovere la continuità assistenziale tra i vari erogatori di prestazioni sanitarie".

Negli Stati Uniti, Edna K. Hufmann, nel suo Medical Record Management [17], fornisce la seguente definizione di "Medical (Health) Record": “A compilation of pertinent facts of a patient's life and health history, including past and present illnesses and treatments, written by the health professionals contributing to the patient's care. The Health Record must be compiled in a timely manner and contain sufficient data to identify the patient, support the diagnosis, justify the treatment and accurately document the results. The primary purpose of the Health Record is to facilitate clinical care and the continuity of that care and it must therefore be easily and quickly retrievable at all times. The record acts as an aide-memoire for the treating clinician and it is also an essential communication tool where other clinicians or healthcare professionals contribute to the care of the patient and it facilitates the patient receiving appropriate treatment at the right time. The secondary purpose of the record is to provide a dependable source of clinical data to support clinical audit, research, teaching, resource allocation and performance planning and the link between the primary and secondary purposes is clinical coding".

Al di là delle definizioni e delle disquisizioni su contenuti, forma, valore giuridico e molto altro ancora, preme rimarcare che si tratta di uno strumento di lavoro, testimone affidabile del modus operandi di singoli professionisti e di intere équipe sanitarie [18].

Molti sono i professionisti, artefici e fruitori della documentazione clinica che ne evidenziano le manchevolezze e manifestano insoddisfazione in proposito. Professionisti che, se da un lato lamentano l'aggravio dell'onere documentale oggi diffusamente richiesto, dall'altro rilevano:

- difficoltà nel rinvenire informazioni ritenute indispensabili, oppure,

- se presenti, difficoltà nel rintracciarle in una pletora - o un coacervo - di scritti;

- difficoltà nel ricostruire l'evoluzione di un quadro clinico o nel cogliere rapidamente i problemi aperti in un determinato momento.

Impostare e gestire i documenti sanitari per andare incontro alle effettive esigenze dei sanitari è senza dubbio un obiettivo sfidante per molteplici ragioni. Basti considerare la pluralità e la diversità delle figure professionali che, intervenendo nel processo assistenziale, hanno titolo a contribuire alla produzione di documenti e quindi ad avvalersene. 
Un primo interrogativo che ci si pone verte pertanto sugli elementi di contenuto che potrebbero essere utili alle diversificate decisioni clinico-assistenziali.

In Francia, nello stilare raccomandazioni sulla formazione del dossier ospedaliero, si suggerì di costituire una base comune di documentazione clinica, prontamente condivisibile da tutti i sanitari, formata da elementi strutturati e ben organizzati in opportune rubriche, e di prevedere l'aggiunta di parti specialistiche, in rapporto alle occorrenze del caso.

Se seguissimo questa impostazione, dovremmo chiederci a chi rimettere la definizione dei contenuti. Non dovrebbero essere le categorie professionali coinvolte a puntualizzare $i$ dettagli informativi, in ragione dei loro peculiari fabbisogni di conoscenza, diversi anche all'interno del gruppo di appartenenza, soprattutto di quello medico?

Con l'affermarsi dei più recenti profili professionali sanitari, la documentazione, per alcuni strumento di assoluta novità, ha finito talvolta per assurgere a emblema di un'autonomia a lungo attesa. Di qui è scaturita una decisa lievitazione di scritture, rispetto alla quale occorre serenamente chiedersi se sia indispensabile per apportare un effettivo miglioramento informativo, giovando così al miglioramento dei processi di cura.

Se l'agire delle diverse figure professionali è sempre più interconnesso, la documentazione, che di quell'agire è specchio, non potrà che improntarsi all'insegna della medesima integrazione. Si dovrebbero evitare, quanto più possibile, le trascrizioni e le ridondanze non utili o, ancor peggio, recanti rilievi non assonanti che, ingenerando incertezze interpretative, rappresentano un pericolo da non sottovalutare.

Da quanto fin qui osservato discende la constatazione che lasciando a ogni gruppo professionale il compito di stabilire indipendentemente $i$ contenuti di interesse, specie in un ospedale polispecialistico, si rischierebbe di pervenire a una proliferazione di modelli abnorme, di difficile gestione e di dubbia funzionalità.

È verosimile supporre che si accentuerebbero, anziché affievolirsi, le difficoltà nel rintracciare le informazioni utili agli uni o agli altri, ancorché presenti, se non ridondanti, nel contesto di una pletora di registrazioni variamente impostate. Di qui l'esigenza di individuare il massimo comune denominatore di contenuto, frutto del paziente raccordo e della mediazione tra le innumerevoli attese delle parti. Stabilita una base informativa trasversale, si dovrebbero poi esaminare attentamente le proposte di aggiunta di elementi specialistici, secondo criteri di stretta indispensabilità, di non eccedenza e di integrazione.

Un altro interrogativo riguarda il modo in cui le informazioni - o parte delle stesse - dovrebbero essere esposte, dal momento che la comprensibilità del contenuto documentale è indubbiamente influenzata anche dalla scelta del metodo espositivo. Il testo libero offre la più ampia flessibilità descrittiva, ma espone a pericoli di fraintendimenti e non facilita un rapido recupero delle informazioni. Le descrizioni standardizzate, tratte da dizionari sanitari, o i codici appartenenti a determinate nomenclature presentano il vantaggio dell'uniformità ma trascinano con sé non poche riserve. L'adozione di sistemi di codifica delle malattie, da cui ci si attenderebbe una spinta verso l'omogeneità, non sempre ha sortito gli sperati effetti.

Consideriamo il percorso logico che dall'osservazione di un paziente riconduce il quadro patologico a voci di una classificazione. Il primo passaggio, dagli elementi cosiddetti obiettivi - seppure sovente oggetto di percezione sensoriale e quindi soggettiva - al giudizio clinico, chiama in causa le conoscenze del singolo professionista e la correttezza della sua valutazione. Il passaggio seguente consiste nell'individuare un descrittore adeguato a sintetizzare l'informazione raccolta. Questo secondo tratto è condizionato dalla granularità del sistema di codifica, dall'accuratezza dei descrittori e dall'ulteriore giudizio del sanitario, chiamato a riportare a sintesi quanto riscontrato.

La capacità di un sistema di codifica di adattarsi alle innumerevoli sfaccettature della realtà, evitando approssimazioni grossolane, contribuisce a misurarne la validità, così come la frequenza degli aggiornamenti, da mantenere al passo con l'approfondirsi delle conoscenze.

Il rischio di perdita di informazioni o di una loro inadeguata rappresentazione dipende da questo intreccio di componenti.

Nello statement elaborato dal Royal College of Physicians (RCP) inglese, e poi adottato dall'Academy of Medical Royal Colleges, nel maggio 2010, si afferma: "The record of the dialogue between the clinician and the patient, the decisions made and the actions taken, is the cornerstone of the patient record. The information that is recorded should be accessible whatever the setting or context. This information can take the form of free text, or of structured data that is completely interoperable, and transferable between clinical applications, contexts and settings without ambiguity. Structured clinical data collected in this way also provides the best source of information for the many purposes that underpin service evaluation and research" [19].

Nel documento dello stesso RCP, An Information Revolution. Consultation response - Question 24 [20], si sostiene: "Capture of data in a structured, standardised form by professionals at the point of care will carry a resource overhead in terms of time and require considerable culture change to achieve widespread acceptance by clinical professionals".

L'adozione di sistemi di gestione informatica ha reso possibile la scrittura di intere proposizioni mediante la composizione di voci selezionate lungo percorsi predefiniti, con vario livello di dettaglio: si ripropone al riguardo la considerazione inerente il livello di dettaglio del dataset da cui il clinico può attingere.

Le rappresentazioni figurative (disegni anatomici ecc.) possono integrare utilmente le scritture, specie in alcuni ambiti, permettendo di cogliere a colpo d'occhio una localizzazione, un'estensione, una morfologia ecc. Il collegamento all'iconografia disponibile (per esempio: referti di diagnostica integrati con le immagini da cui sono desunti) consente un approfondimento immediato, senza bisogno di attività di recupero della fonte primaria.

Per facilitare le scelte clinico-assistenziali, è importante che ogni componente documentale sia corredato dagli estremi identificativi di chi ha compiuto un'azione o ha assunto una decisione e dalle coordinate temporali degli eventi descritti (data e, possibilmente, ora e minuti), imprescindibili per permettere una correlazione puntuale con l'andamento delle condizioni dell'assistito.

Una documentazione che intenda essere funzionale al decision making dovrebbe riferire anche le motivazioni delle 
scelte compiute, dei trattamenti disposti, attuati, sospesi o revocati.

La dottrina [21] ha affermato la rilevanza dell'argomentare l'iter logico seguito dal professionista, a partire dai presupposti considerati, con un'enunciazione accurata dei passaggi chiave e pure di suoi eventuali ripensamenti. Anche nella regolamentazione documentale francese si è evidenziata la necessità che, in occasione di ogni modificazione terapeutica, il medico lasci traccia della relativa motivazione, data l'utilità di comprendere la ragione delle decisioni adottate.

Quando le cure di un paziente si protraggono nel tempo, il fascicolo sanitario è solitamente corposo; per facilitarne la consultazione e permettere di cogliere prontamente problemi e trattamenti in corso, assumono importanza le valutazioni cliniche periodiche, per le quali dovranno essere definite cadenze, modalità e responsabilità di attuazione. In relazione alla complessità delle cure da prestare, potrebbe essere opportuno che le sintesi accennate fossero prodotte dalle principali figure professionali coinvolte, sempre in chiave di stretta integrazione. L'esposizione di fatti sopraggiunti, la rilevazione di elementi non previamente riscontrati, la registrazione di nuovi giudizi clinici ecc. dovrebbero poi avvenire in predeterminati settori del fascicolo sanitario, in sequenza con precedenti, analoghe annotazioni, per consentire di cogliere rapidamente l'evoluzione dello stato del paziente.

Una disposizione razionale dei documenti e una gestione ordinata sono ulteriori fattori che possono semplificare la ricerca di informazioni cliniche: obiettivo il cui raggiungimento è favorito anche da un'impostazione standardizzata ed ergonomica del layout documentale.

Da gran tempo i sanitari invocano l'avvento dell'informatica per superare una serie di ostacoli che ancora si frappongono a una fluida, organica fruizione dei dati sanitari. Le esperienze di informatizzazione delle principali gestioni sanitarie, peraltro, non si sono sempre rivelate soddisfacenti e, quel che più preoccupa, rispondenti ai canoni di una corretta e sicura gestione documentale. I requisiti esigibili da un documento sanitario devono trovare piena applicazione anche nelle versioni elettroniche, seppure con gli adattamenti richiesti dal diverso strumento, e particolare attenzione va riservata alle gestioni miste - cartacea e informatica - per garantire coerenza e certezza delle informazioni veicolate. Oltre gli indubbi benefici all'operatività quotidiana, una gestione informatica, per i rischi connessi, richiede garanzie di business continuity, integrità, security, privacy dei dati e definizione di regole per la gestione delle bozze, delle correzioni, della produzione di stampe aggiornate di supporto e così via.

A proposito di gestione informatica dei record sanitari, appare di interesse quanto evidenziato, ai primi del 2011, dal RCP nel citato documento An Information Revolution. Consultation response [20]: un'attenzione troppo accentuata sui dati gestionali ha portato a un disimpegno sul versante dei dati clinici, limitando così la validità, la veridicità e l'utilità delle informazioni raccolte.

Oltre la trattazione informatica dei singoli documenti, sta avanzando la realizzazione del Fascicolo Sanitario Elettronico di ogni persona che ne voglia consentire la creazione. La documentazione di ricovero è destinata a confluirvi, anche se inizialmente in modalità sintetica (eventi di ingresso, uscita, lettera di dimissione, verbale di Pronto Soccorso). Rilevanti le ricadute positive per i clinici, quanto a conoscenza dei precedenti sanitari di una persona, pur tenendo presenti le limitazioni derivanti da eventuali scelte di "oscuramento" dettate da tutela della riservatezza.

Le prospettive dischiuse dal Codice della Pubblica Amministrazione Digitale (D.Lgs 82/2005 e successive modificazioni, in particolare la conservazione sostitutiva di una serie di documenti) hanno spianato la strada alla realizzazione di poli archivistici sanitari digitalizzati: nuovi, fecondi giacimenti di saperi disponibili on line, a seguito del riversamento di documenti originariamente informatici o convertiti a partire da originali. In proposito si cita, per esempio, il Polo Archivistico della Regione Emilia Romagna (PARER, http: / / parer.ibc.regione.emilia-romagna.it/).

\section{Conclusione}

Per migliorare la disponibilità di informazioni, snellire il lavoro dei sanitari e favorirne i processi decisionali, occorre una riconsiderazione estesa e approfondita dell'intera cartella clinica: lavoro impegnativo che può sortire positivi risultati solo a condizione che vi sia una convinta partecipazione delle molteplici professionalità interessate.

\section{Conflitto di interessi}

Gli autori dichiarano di non aver nessun conflitto di interessi.

\section{Bibliografia}

[1] Negrini G, La Pietra L. Documentazione sanitaria: come evitare la deriva protezionistica? Professione \& Clinical Governance 2009;7:22-3.

[2] Bacci M. La cartella clinica. In: Barni M, Greco M, Malagnino G, Meledandri B, Paci A, Panti A (a cura di). Guida all'esercizio professionale per i medici chirurghi e gli odontoiatri. Torino: CG Edizioni Medico Scientifiche, 2000.

[3] Braga $M$, Nonis $M$. Informazione e cartella clinica. In: Nonis $M$, Braga M, Guzzanti E (a cura di). Cartella clinica e qualità dell'assistenza. Roma: Il Pensiero Scientifico, 1998.

[4] Buzzi F, Sclavi C. La cartella clinica: atto pubblico, scrittura privata o tertium genus. Riv It Med Leg 1997;4:1161.

[5] Candia L, Cambieri A. La cartella clinica: strumento di lavoro e di studio, mezzo di prova. Medicina e Morale 1991;4:569-83.

[6] Del Vecchio S, Gasparrini V, Lelli S, Martelloni M, Puntoni G, Ricci M. La cartella clinica e la cartella infermieristica. In: Strumenti di management e indicatori di qualità delle prestazioni sanitarie. Torino: CG Edizioni Medico Scientifiche; 2006.

[7] Gattai A. Cartella clinica. Milano: OEMF; 1979.

[8] Giuliani P, Negrini G, Alborghetti A, Di Biasi C, Casati M, Vimercati F, et al. Manuale della cartella clinica, II Ed, Regione Lombardia; 2007.

[9] Guzzanti E, Tripodi G. L'ospedale e la medicina sociale. Roma: IMS; 1996.

[10] Merli S, Ciallella C. Interesse e attualità della cartella clinica. Roma: Zacchia; 1992. p. 255-73.

[11] Merusi F, Bargagna M. La cartella clinica. Milano: Giuffrè; 1978.

[12] Monza G, Mantovani R, Perelli Ercolini M. La cartella clinica ospedaliera. Milano: Health Communication; 2005. 
[13] Negrini G, La Pietra L. Documentazione sanitaria. Guida all'esplorazione di un universo variegato, verso nuove. sfidanti mete: dalla cartella di ricovero alle molteplici altre forme documentali. Milano: Tecniche Nuove; 2011.

[14] Negrini G. Il dossier clinico multimediale. Professione \& Clinical Governance 2008;2:4-8.

[15] www.legifrance.gouv. fr/affichCode.do?cidTexte=LEGITEXT000006072665\&dateTexte $=20080314$.

[16] Joint Commission International. Accreditation Standards for Hospitals, $4^{\text {th }}$ Ed, IL: Oakbrook Terrace; 2010.
[17] Huffman EK. Medical Record Management. Physicians Record Company: Chicago, IL; 1990.

[18] Negrini G. Una documentazione clinica al servizio del decision making. Professione \& Clinical Governance 2009;7:27-30.

[19] www.rcplondon.ac.uk/clinical-standards/hiu/medical-records.

[20] www.rcplondon.ac.uk/sites/default/files/rcp-responseto-information-revolution-consultation-14-jan-2011.pdf.

[21] Pagni A, Fucci S (a cura di). Manuale della professione medica. Deontologia etica normativa. Torino: CG Edizioni Medico Scientifiche, 2011. 